OPEN

SUBJECT AREAS:

CANCER

MICROENVIRONMENT

TUMOUR ANGIOGENESIS

BREAST CANCER

Received

11 April 2014

Accepted

9 July 2014

Published

28 July 2014

Correspondence and requests for materials should be addressed to A.S.P. (apopel@jhu. edu)

\section{Lymphatic endothelial cells support tumor growth in breast cancer}

\author{
Esak Lee' , Niranjan B. Pandey' \& Aleksander S. Popel ${ }^{1,2}$
} 'Department of Biomedical Engineering, Johns Hopkins University School of Medicine, Baltimore, MD 21205 , United States,
2Department of Oncology and the Sidney Kimmel Comprehensive Cancer Center, Johns Hopkins University School of Medicine,
Baltimore, MD 21231, United States.

Tumor lymphatic vessels (LV) serve as a conduit of tumor cell dissemination, due to their leaky nature and secretion of tumor-recruiting factors. Though lymphatic endothelial cells (LEC) lining the LV express distinct factors (also called lymphangiocrine factors), these factors and their roles in the tumor microenvironment are not well understood. Here we employ LEC, microvascular endothelial cells (MEC), and human umbilical vein endothelial cells (HUVEC) cultured in triple-negative MDA-MB-231 tumor-conditioned media (TCM) to determine the factors that may be secreted by various EC in the MDA-MB-231 breast tumor. These factors will serve as endothelium derived signaling molecules in the tumor microenvironment. We co-injected these EC with MDA-MB-231 breast cancer cells into animals and showed that LEC support tumor growth, HUVEC have no significant effect on tumor growth, whereas MEC suppress it. Focusing on LEC-mediated tumor growth, we discovered that TCM-treated LEC ('tumor-educated LEC') secrete high amounts of EGF and PDGF-BB, compared to normal LEC. LEC-secreted EGF promotes tumor cell proliferation. LEC-secreted PDGF-BB induces pericyte infiltration and angiogenesis. These lymphangiocrine factors may support tumor growth in the tumor microenvironment. This study shows that LV serve a novel role in the tumor microenvironment apart from their classical role as conduits of metastasis.

$\mathrm{T}$ he tumor microenvironment is an emerging target to treat tumor growth and metastasis ${ }^{1}$. The tumor microenvironment comprises cancer cells, extracellular matrix (ECM), and non-cancer stromal cells. The non-cancer stromal cells are more stable and targetable, compared to cancer cells, as the cancer cells experience genetic mutations and epigenetic alterations, resulting in heterogeneity and plasticity ${ }^{2}$. Importantly, these stromal cells contribute to cancer progression through their complex crosstalk with cancer cells. For example, certain types of immune cells, such as tumor-associated macrophages (TAM) accelerate tumor angiogenesis and tumor growth by overexpressing angiogenic and tumor promoting factors in the tumor microenvironment ${ }^{3}$. Some mesenchymal cell types, such as cancer-associated fibroblasts (CAF) also induce tumor growth, epithelial to mesenchymal transition (EMT), and metastasis through their secreted factors ${ }^{4,5}$.

The other important component in the tumor microenvironment is the blood and lymphatic vasculature. Blood and lymphatic vessels are formed in and around the tumor via the angiogenic and lymphangiogenic cues derived from cancer cells and stromal cells ${ }^{6,7}$. Tumor blood vessels are well recognized as key players in tumor growth, because growing tumor cells require oxygen and nutrients via the blood supply into the tumor ${ }^{8}$. More recently studies detailing the crosstalk between cancer cells and blood endothelial cells (BEC) via the BECsecreted proteins (also called angiocrine factors) ${ }^{9-16}$ have helped to change the perspective on the role of the blood vasculature from that of a passive conduit to a more inductive role.

Lymphatic vessels (LV) in the tumor microenvironment are primarily considered as a route of tumor dissemination ${ }^{17}$. LV are leaky compared to blood vessels, because they are only sparsely covered with pericytes and smooth muscle cells ${ }^{18}$. Thus in certain types of cancers such as in breast cancer, metastasis occurs preferentially through the lymphatic vasculature compared to the blood vessel mediated spreading ${ }^{19,20}$. LEC show distinct gene expression profiles from BEC ${ }^{21}$. However, LEC-secreted proteins (also called lymphangiocrine factors) in tumor microenvironment and their crosstalk with cancer cells are poorly understood compared to BEC-derived angiocrine factors. Thus an understanding of the lymphangiocrine factors would add to the current understanding of the role of the LV in lymphatic dissemination of tumor cells ${ }^{22}$.

We previously reported on pro-metastatic crosstalk between breast cancer cells and LEC. We showed that LEC within pre-metastatic lungs and lymph nodes are enhanced and conditioned by triple-negative breast cancer (TNBC) cell secretions and promote spontaneous metastases within one month ${ }^{23}$. Here we investigate roles of 
LEC in the tumor microenvironment in primary tumor growth and pericyte recruitment by employing in vitro tumor-educated LEC models and in vivo LEC-included breast tumor xenograft and matrigel plug models. This study shows for the first time that LEC can support tumor growth through lymphangiocrine factors.

\section{Results}

LEC within tumor microenvironment promote tumor growth. We co-injected EC (LEC, MEC, and HUVEC) with MDA-MB-231 breast cancer cells into animals. In a conventional MDA-MB-231 (MB231) breast tumor xenograft model, two million MB231 cancer cells are orthotopically inoculated with $50 \%$ matrigel supplement. In this study, however, the conventional xenograft model was modified as follows: the number of cancer cells was decreased to 300,000 MB231 cells; a large number of LEC or MEC or HUVEC (2,000,000 cells) were included with the cancer cells, consistent with the procedures employed in several previous studies ${ }^{24-27}$. Matrigel was not added in the tumor/EC mixture to remove matrigel effects: in general, matrigel significantly promotes primary tumor establishment and growth $^{28}$. This new xenograft model slows down tumor growth rates, compared to the normal xenograft model; at the same time it gives a better understanding of roles of EC in tumor growth. There were seven experimental arms: $300,000 \mathrm{MB} 231$ only $(\mathrm{N}=8)$; $300,000 \mathrm{MB} 231+2,000,000$ LEC $(\mathrm{N}=8) ; 300,000 \mathrm{MB} 231+$ 2,000,000 MEC $(\mathrm{N}=8) ; 300,000 \mathrm{MB} 231+2,000,000$ HUVEC $(\mathrm{N}$ $=8) ; 2,000,000$ LEC only $(\mathrm{N}=2) ; 2,000,000$ MEC only $(\mathrm{N}=2)$; and $2,000,000$ HUVEC only $(\mathrm{N}=2)$. Total volume of each injection was identical. LEC-included MB231 tumors showed dramatic increases in the tumor growth rate, compared to tumors containing only MB231 cells. Interestingly, MEC-included MB231 tumor showed suppression of tumor growth. HUVEC-included MB231 tumors did not show any difference compared to MB231 tumors (Fig. 1a,b).

LEC show distinctive secretomes after tumor education with TCM. Having the results shown in Figure $1 \mathrm{a}$ and $1 \mathrm{~b}$, we hypothesized that LEC, MEC and HUVEC would secrete different factors in response to MB231 tumor-conditioned media (TCM) and these factors could differentially affect tumor growth. To test this hypothesis, tumor-educated LEC (LEC cultured in TCM, also called MB231-LEC), tumor-educated MEC (MB231-MEC), and tumor-educated HUVEC (MB231-HUVEC) were prepared by the process described in the Supplementary Fig. S1 online. The tumoreducated conditioned media from these tumor-educated EC were compared with normal conditioned media obtained from normal LEC, MEC, and HUVEC to show how the TCM affects the various endothelial secretomes (Fig. 1c,d). A Proteome Profiler Antibody Arrays Kit for Human Angiogenesis (R\&D systems, Minneapolis, $\mathrm{MN}$ ) was used to determine 55 angiogenesis-related factors in these conditioned media (Fig. 1c). Average pixel number of the pair of duplicate spots was determined and the \% changes in protein levels were plotted. Changes in protein levels more than $200 \%$ between normal and tumor-induced groups were considered to be significant. After TCM education, LEC showed large increases in EGF (>608\%), PDGF-BB ( $>281 \%)$, and VEGF $(>600 \%)$; MEC showed large increases in GM-CSF $(>810 \%)$, CD26 (>580\%), and HB-EGF (210\%); however HUVEC did not show any dramatic changes $(<200 \%)$ (Fig. 1d). As LEC have shown enhanced tumor growth (Fig. 1a,b), we focused on LEC secretome (EGF, PDGF-BB, and VEGF). A previous experiment with the same antibody array showed that MB231 TCM contains Angiogenin, CXCL16, Endothelin-1, HB-EGF, IL-8, MMP-8, MMP-9, PDGF-AA, PlGF, and VEGF ${ }^{29}$. Hence, we excluded VEGF in the LEC secretome and further studied EGF and PDGF-BB, the factors that are not expressed by MB231 cells.

MB231-LEC express high amounts of EGF which induces cancer cell proliferation. An ELISA for human EGF was performed with
MB231-LEC, MB231-HUVEC, and normal LEC conditioned media (Fig. 2a). hEGF was highly expressed in MB231-LEC compared to other groups (Fig. 2a). We estimated that 1,000 MB231-LEC cells expressed $1.1 \pm 0.16 \mathrm{pg}$ hEGF. MB231 cell proliferation was assessed in MB231-LEC conditioned media (MB231-LEC CM), MB231HUVEC CM, normal LEC CM, and serum-free media (Fig. 2b). Only the MB231-LEC CM potently promoted MB231 cell proliferation (Fig. 2b). Next, MB231 cell proliferation in the MB231LEC CM was assessed with anti-EGFR neutralizing antibodies (Fig. 2c). Anti-EGFR antibodies blocked MB231-LEC induced MB231 cell proliferation (Fig. 2c). Confluent LEC were treated with control media (30\% SFM $+70 \% \mathrm{EGM})$ or $30 \%$ TCM media (30\% TCM $+70 \%$ EGM) overnight (Fig. 2d). When LEC were incubated with $30 \%$ TCM, LEC expressed hEGF, however, normal LEC in the normal media did not express hEGF (Fig. 2d). We further studied hEGF expression in LEC in a LEC/MB231 co-culture model (Fig. 2e). We mixed LEC and MB231 (1:1 ratio) in 50\% EGM and $50 \%$ RPMI-1640 media and make the cell adhere on the bottom of plates overnight. We then replaced the co-media with serum-free media and incubated cells overnight. We fixed the cells and stained them with antibodies for hProx-1 (LEC marker) and hEGF (Fig. 2e). In the co-culture condition, the Prox-1 positive LEC were colocalized with hEGF, demonstrating lymphatic expression of hEGF in tumor/ LEC co-culture models (Fig. 2e). Mice with LEC-included matrigel plugs were treated with TCM or SFM for 10 days as described in Supplementary Fig. S2 online. The excised gel plugs were analyzed with anti-hProx-1 and anti-hEGF antibodies. Colocalization of hProx-1 and hEGF only in the TCM-treated LEC/matrigel plugs, not in the SFM-treated group, suggests that LEC secrete EGF when exposed to tumor-secreted factors (Fig. 2f).

LEC are associated with tumor cell proliferation. Upon the discovery that EGF expressed by MB231-LEC induces MB231 cancer cell proliferation (Fig. 2), we analyzed LEC-included MB231 tumors and normal MB231 tumors by immunostaining them with anti-human VEGFR3 (a lymphatic marker) and antihuman PCNA (proliferating-cell nuclear antigen) antibodies to detect human LEC and proliferating tumor cells (Fig. 3a). We observed that LEC-included tumors had more prevalent human PCNA-positive cells around hVEGFR3-positive LEC areas, compared to normal MB231 tumors. However, normal MB231 tumors showed less PCNA-positive cells in the intratumoral area, and the proliferating cells were mostly detected in the peritumoral areas (Fig. 3a,b). The results suggest that LEC may facilitate tumor cell proliferation in the tumor microenvironment. To confirm the proliferating human cells are not LEC, but MB231 cells, we further observed the LEC-included MB231 tumor sample in a high resolution (Fig. 3c). Human VEGFR3-positive LEC (red) were not colocalized with pEGFR (yellow, pseudo-colored) and hPCNA (green), but other human cells adjacent to LEC (supposed to be MB231 cancer cells) showed pEGFR and hPCNA signals (Fig. 3c). This demonstrates that LEC-secreted EGF (Fig. 2) can phosphorylate EGFR in the LEC-adjacent MB231 cells and induce MB231 cell proliferation however autocrine effects of the LEC-secreted EGF in LEC proliferation was not detected (Fig. 3c).

MB231-LEC express high amounts of PDGF-BB and recruit pericytes in vivo. Two million LEC or HUVEC included matrigels were implanted in athymic nude mice and the mice were subcutaneously treated with $50 \mu \mathrm{l}$ TCM or SFM for 10 days. A no-cell group was included as a control group. Ten days after the matrigel implantation, the plugs were excised and analyzed with anti- $\alpha$ SMA (alpha smooth muscle actin) and anti-desmin antibodies, as $\alpha$ SMA and desmin are markers for pericytes ${ }^{30,31}$. LEC-included matrigel from TCM treated animals ('LEC-TCM' group) showed dramatic recruitment of $\alpha$ SMA-positive cells whereas HUVEC-TCM, LECSFM and No cell-TCM groups showed fewer $\alpha$ SMA-positive cells 
a

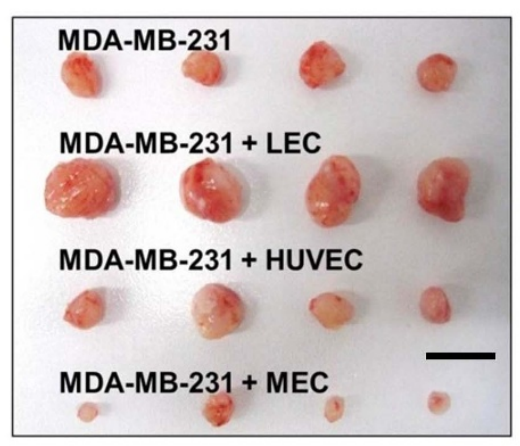

b

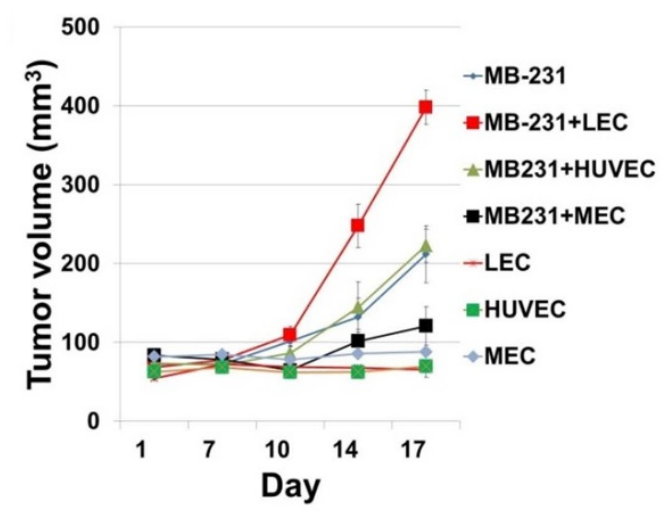

LEC conditioned media (CM)

C
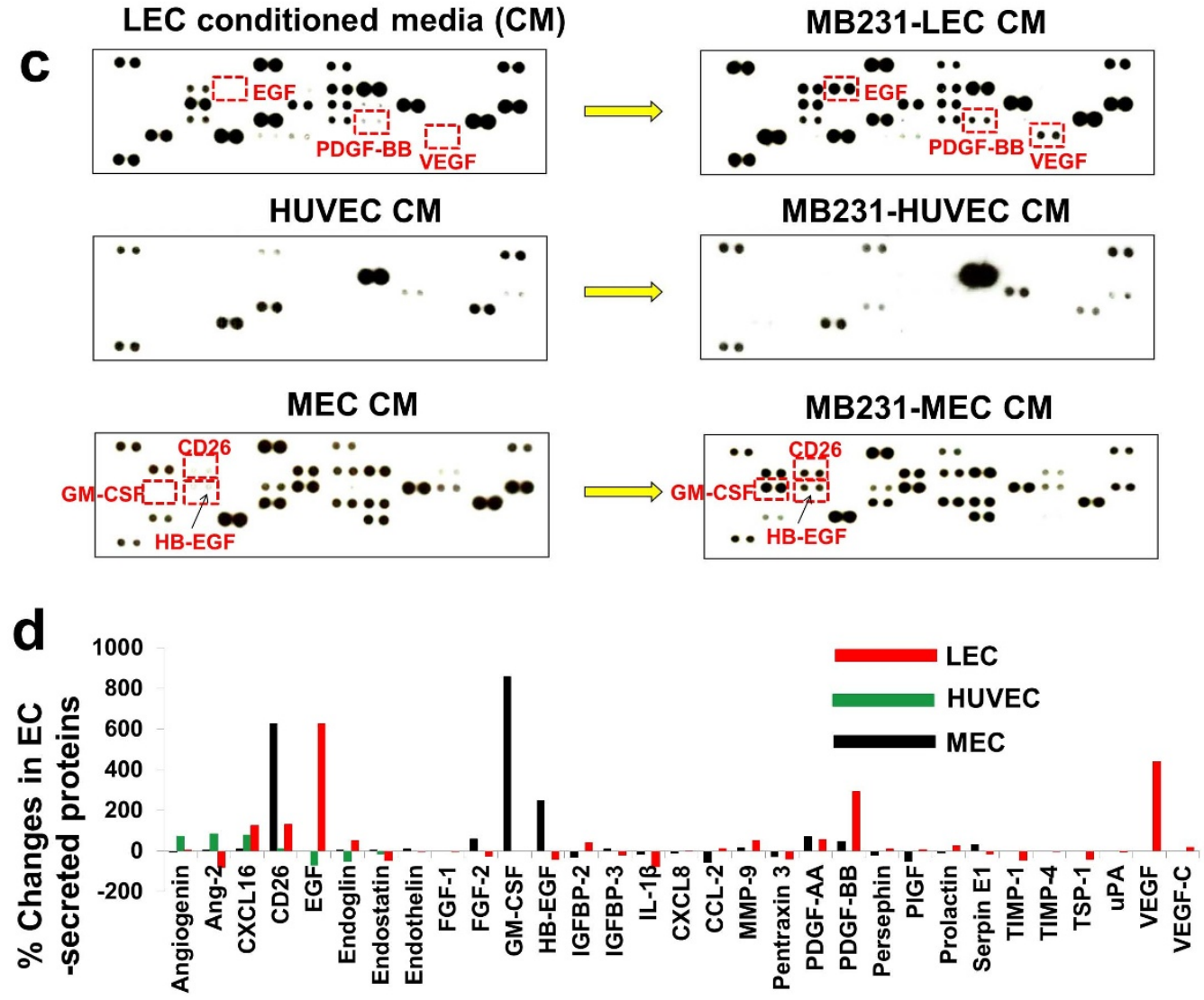

Figure $1 \mid$ LEC promote tumor growth and show distinctive secretomes under TCM condition. (a) Two million of LEC, HUVEC, or MEC were premixed with 300,000 MB231 cells respectively, after which the cell mixtures were orthotopically inoculated into the thoracic part $\left(2^{\text {nd }}\right.$ mammary site from the top) of athymic nude mice without supplementing with matrigel $(\mathrm{N}=8)$. For control, just 300,000 MB231 ( $\mathrm{N}=8$ ) or just 2,000,000 EC (LEC; MEC; HUVEC, $\mathrm{N}=2$ each EC) were inoculated. Representative tumors in each group, scale bar represents $10 \mathrm{~mm}$. (b) Tumor growth curve. (c) Fifty five angiogenesis-related factors from tumor-educated LEC/MEC/HUVEC (MB231-LEC/MB231-MEC/MB231-HUVEC) were examined by using reverse western arrays. Factors secreted from LEC, MEC, and HUVEC before and after incubating them in 30\% TCM were analyzed and compared. MB231-LEC shows high expression of EGF, VEGF and PDGF-BB, compared to normal LEC. MB231-MEC shows high expression in CD26, HB-EGF and GM-CSF, compared to normal MEC. HUVEC does not show dramatic changes. (d) \% Changes in EC secreted proteins. Average pixel density of the pair of duplicate spots was determined by using the ImageJ. Expression level changes more than $200 \%$ after TCM treatment were considered as significant. Data (b) are reported as mean \pm s.e.m.

(Fig. 4a,b). Anti-desmin (red) and anti-mCD31 (green) antibodies were employed to assess the pericyte coverage on the blood vessels (Fig. 4c). Anti-hVEGFR3 antibodies (blue) were also used to detect human LEC initially loaded in the gel plugs. The pericyte coverage was more frequently observed in LEC-matrigel plugs from mice that had been treated with TCM than other groups, including 'HUVECTCM', 'LEC-SFM', and 'No cell-TCM' groups (Fig. 4c). We also demonstrated that anti- $\alpha \mathrm{SMA}$ and anti-desmin antibodies are not immunoreactive with human VEGFR3-positive LEC (blue) or mouse CD31-positive BEC (green) in the LEC-SFM control, suggesting that the anti- $\alpha \mathrm{SMA}$ and anti-desmin antibodies are pericyte specific (Supplementary Fig. S3 online).

PDGF-BB in MB231-LEC conditioned media (MB231-LEC CM) was measured by ELISAs, comparing it with normal LEC CM, 

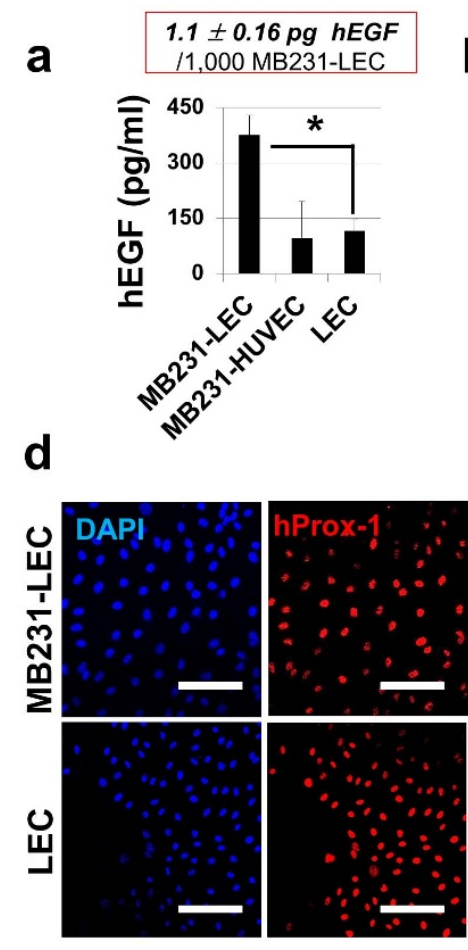

Monoculture (LEC)


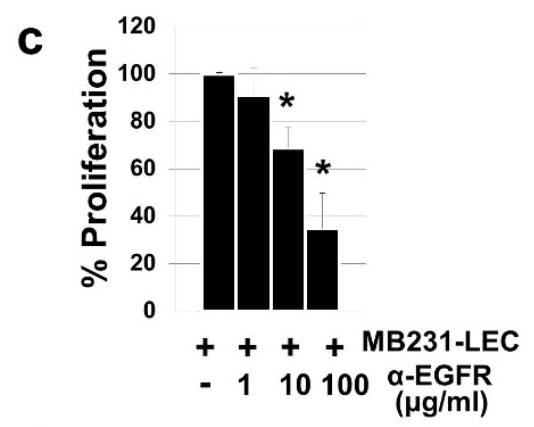

e
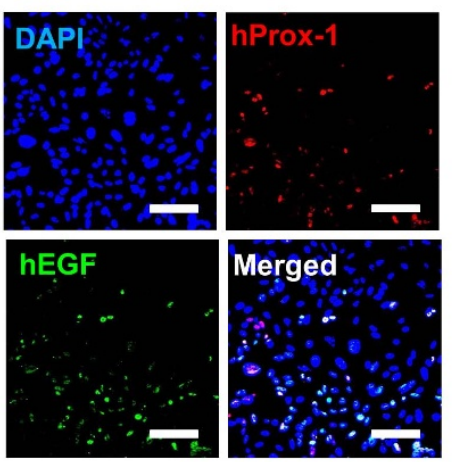

Co-culture (LEC + MB231)

\section{f}
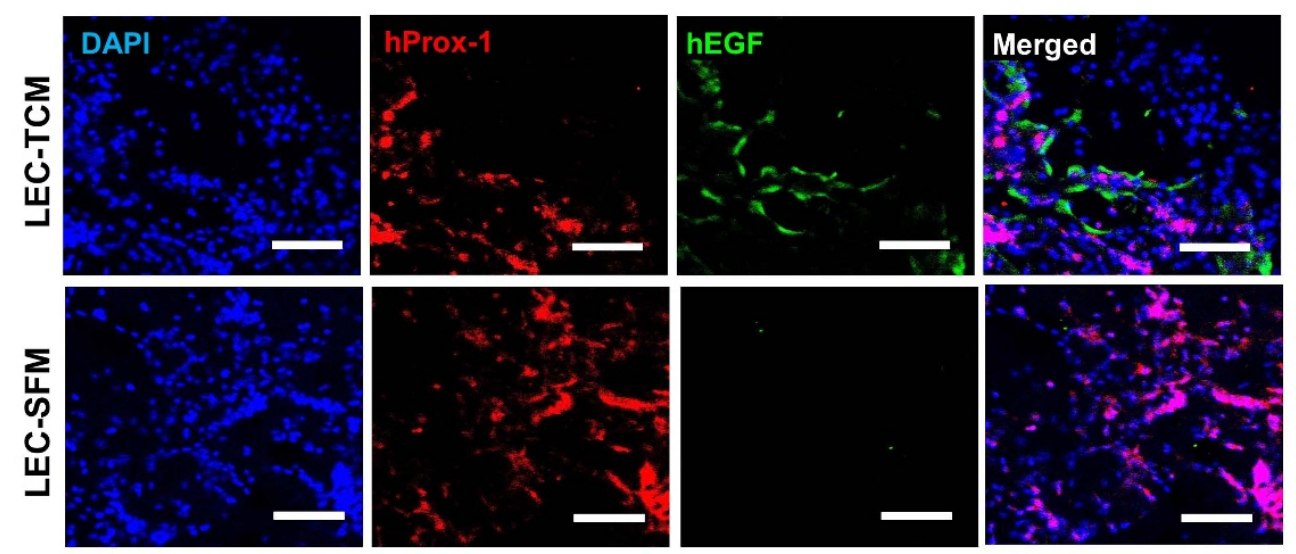

Figure $2 \mid$ MB231-LEC express EGF and induce cancer cell proliferation. (a) ELISA for human EGF on MB231-LEC, MB231-HUVEC, and normal LEC conditioned media. MB231-LEC express high amount of EGF compared to normal LEC $(* \mathrm{P}=0.042)$. We estimate that $1,000 \mathrm{MB} 231$-LEC cells expressed $1.1 \pm 0.16$ pg hEGF. (b) MB231 cell proliferation was assessed in MB231-LEC CM, MB231-HUVEC CM, normal LEC CM, and serum-free media. MB231-LEC CM significantly promotes MB231 cell proliferation, compared to normal LEC CM ( $\left.{ }^{*} \mathrm{P}=0.040\right)$. (c) MB231 cell proliferation induced by MB231-LEC is blocked by using anti-EGFR antibodies ( ${ }^{*} \mathrm{P}<0.05$ ). (d) Normal LEC in normal EGM media ('LEC') or LEC in TCM ('MB231-LEC') show distinct hEGF expression. Normal LEC do not express hEGF, but TCM-conditioned LEC express hEGF. Scale bars represent $100 \mu \mathrm{m}$. (e) LEC and MB231 were cultured in the same plate, showing the LEC/MB231 co-culture effects. In the co-culture condition, LEC start to express hEGF, however MB231 do not show hEGF expression. Scale bars represent $100 \mu \mathrm{m}$. (f) LEC-included matrigel plugs from TCM or SFM-treated athymic nude mice were analyzed 10 days after matrigel implantation. Human Prox-1 positive LEC in the matrigel plugs express hEGF when the matrigel-implanted animals are treated with TCM ('LEC-TCM' group). LEC from SFM-treated animals do not express hEGF ('LEC-SFM' group). Scale bars represent $100 \mu \mathrm{m}$. Data (a,b,c) are reported as mean \pm s.e.m.

MB231-HUVEC CM, and serum-free media (Fig. 5a). MB231-LEC express high amounts of PDGF-BB compared to the other groups (Fig. 5a). We estimated that 1,000 MB231-LEC cells expressed 2.2 \pm 0.21 pg PDGF-BB. Angiogenesis reverse array data (Fig. 1) showed that EGF increased around $600 \%$, PDGF-BB increased around $280 \%$ in MB231-LEC, compared to normal LEC. As the change in PDGF$\mathrm{BB}$ was relatively smaller than EGF, we additionally performed quantitative RT-PCR for gene expression of $h P D G F B$ in LEC to account for the protein expression data. Approximately 3.4 times more enhanced $h P D G F B$ gene expression was observed in MB231LEC (Supplementary Fig. S4 online). Confluent LEC were treated with control media (30\% SFM $+70 \% \mathrm{EGM})$ or $30 \%$ TCM media (30\% TCM $+70 \%$ EGM) overnight (Fig. $5 \mathrm{~b})$. When only LEC were treated with TCM, LEC expressed hPDGF-B (Fig. 5b). In LEC/ MB231 co-culture model, we also showed that hProx-1 positive LEC (red) express hPDGF-B (green) (Fig. 5c). Mice with LECincluded matrigel plugs were treated with TCM or SFM. LECincluded matrigel from TCM treated animals ('LEC-TCM') showed 

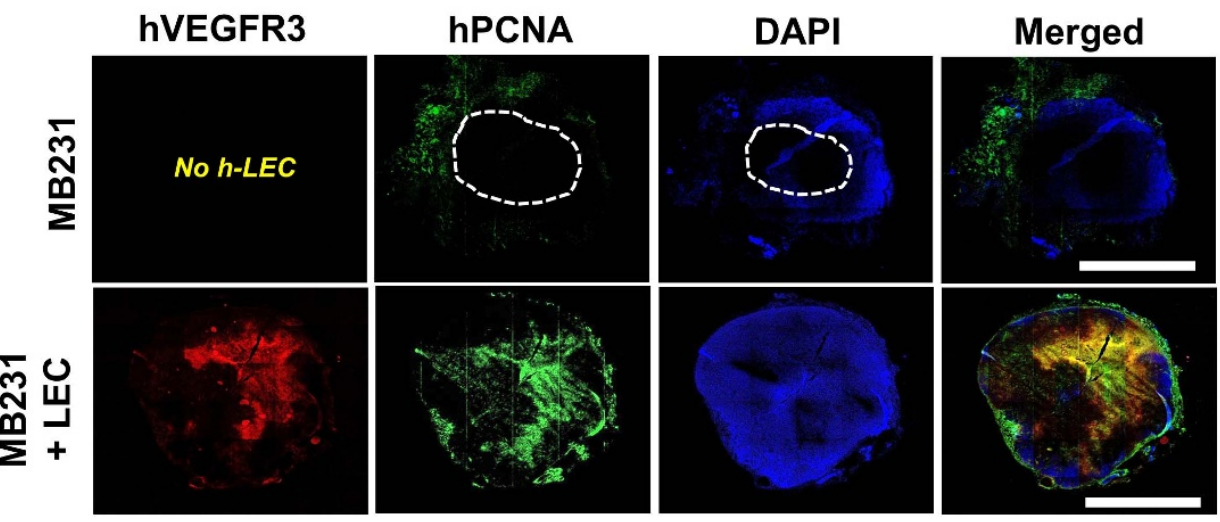

b
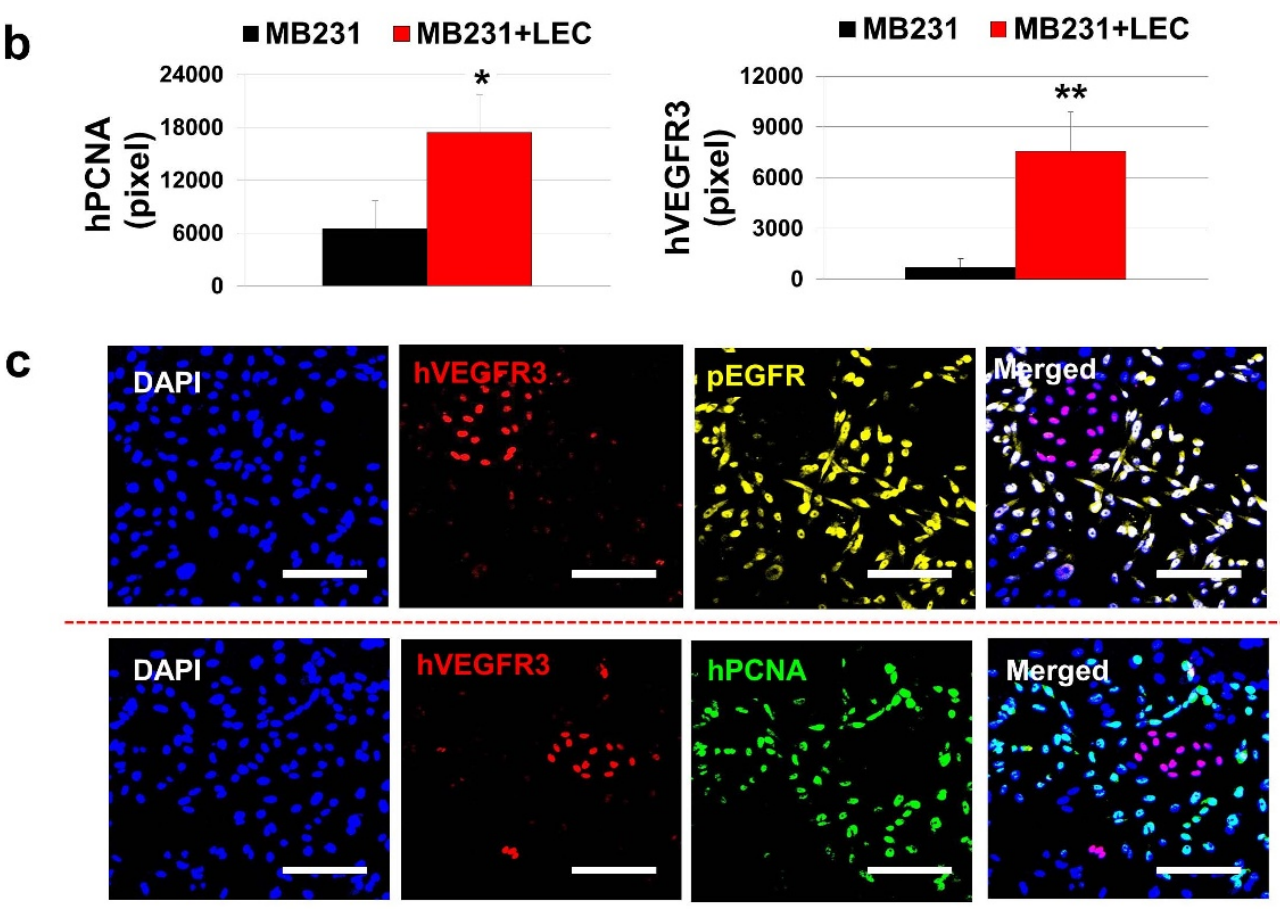

Figure 3 LEC support MB231 cell proliferation in tumor xenografts. (a) Two million LEC were pre-mixed with 300,000 MB231 cells, then, the cell mixtures were orthotopically inoculated into the thoracic mammary fat pad. For control, just 300,000 MB231 were inoculated. Human PCNA positive area representing proliferating human cells were colocalized with LEC in the LEC-included tumors, suggesting that LEC may support tumor cell proliferation. Scale bars represent $4 \mathrm{~mm}$. (b) Quantification of hPCNA $(* \mathrm{P}=0.038)$ and hVEGFR3 (**P $=0.008)$ in the tumor samples. Twelve randomly selected $10 \times$ images (per group) were obtained and the labeled antigens were quantified by using the ImageJ. (c) Proliferating human cells might be either human MB231 or human LEC. Further observation of the LEC-included tumor in a high resolution confirms that the proliferating human cells are human MB231 cells, not the human LEC. Human VEGFR3-positive LEC (red) were not colocalized with pEGFR (yellow, pseudo-colored) and hPCNA (green), but other human cells adjacent to LEC that are supposed to be human MB231 cells, show pEGFR and PCNA signals. Scale bars represent $100 \mu \mathrm{m}$. Data (b) are reported as mean \pm s.e.m.

human PDGF-B expression around human LEC (hProx-1), compared to 'LEC-SFM' matrigel plugs, demonstrating that tumoreducated LEC express hPDGF-B in vivo (Fig. 5d).

SU16f, a PDGFR $\beta$ inhibitor blocks MB231-LEC induced pericyte recruitment and angiogenesis. We further performed LECincluded matrigel plug assays with a PDGFR $\beta$ inhibitor to address the role of LEC-derived PDGF-BB in the model (Fig. 6). We employed SU16f, a PDGFR $\beta$ inhibitor. SU16f selectively inhibits PDGFR $\beta$ over VEGFR2, FGFR1, and EGFR more than 14-fold, 229 -fold and 10000-fold, respectively. This information was provided by Tocris Biosciences, a provider of the SU16f (Catalog No, 3304). Nine animals with LEC-included matrigel plugs (two plugs per mouse) were treated with SFM (3 mice) or TCM (3 mice) or TCM + SU16f (3 mice). Fifty microliter of SFM or TCM were administered subcutaneously every day; $10 \mathrm{mg} / \mathrm{kg}$ SU16f were additionally administered orally every day for the TCM + SU16f group. After 10 days, matrigel plugs were excised and analyzed. Macroscopic images for the gel plugs indicated that the LEC-TCM group experienced more intra-gel angiogenesis compared to other groups (Fig. 6a). We stained the plugs with anti- $\alpha$ SMA and antimCD31 antibodies to detect pericytes and blood vessels (Fig. 6b,c). The LEC-TCM group showed enhanced pericyte recruitment, and angiogenesis, compared to LEC-SFM (Fig. 6b). SU16f treatment significantly inhibits LEC-TCM induced pericyte infiltration and angiogenesis (Fig. 6b,c). The inhibition of PDGFR $\beta$ upon TCM induction by using SU16f and the decreases in pericyte recruitment and angiogenesis demonstrate that MB231-LEC secreted PDGF-BB induce pericyte recruitment and angiogenesis via the PDGFR $\beta$ signaling pathway. 


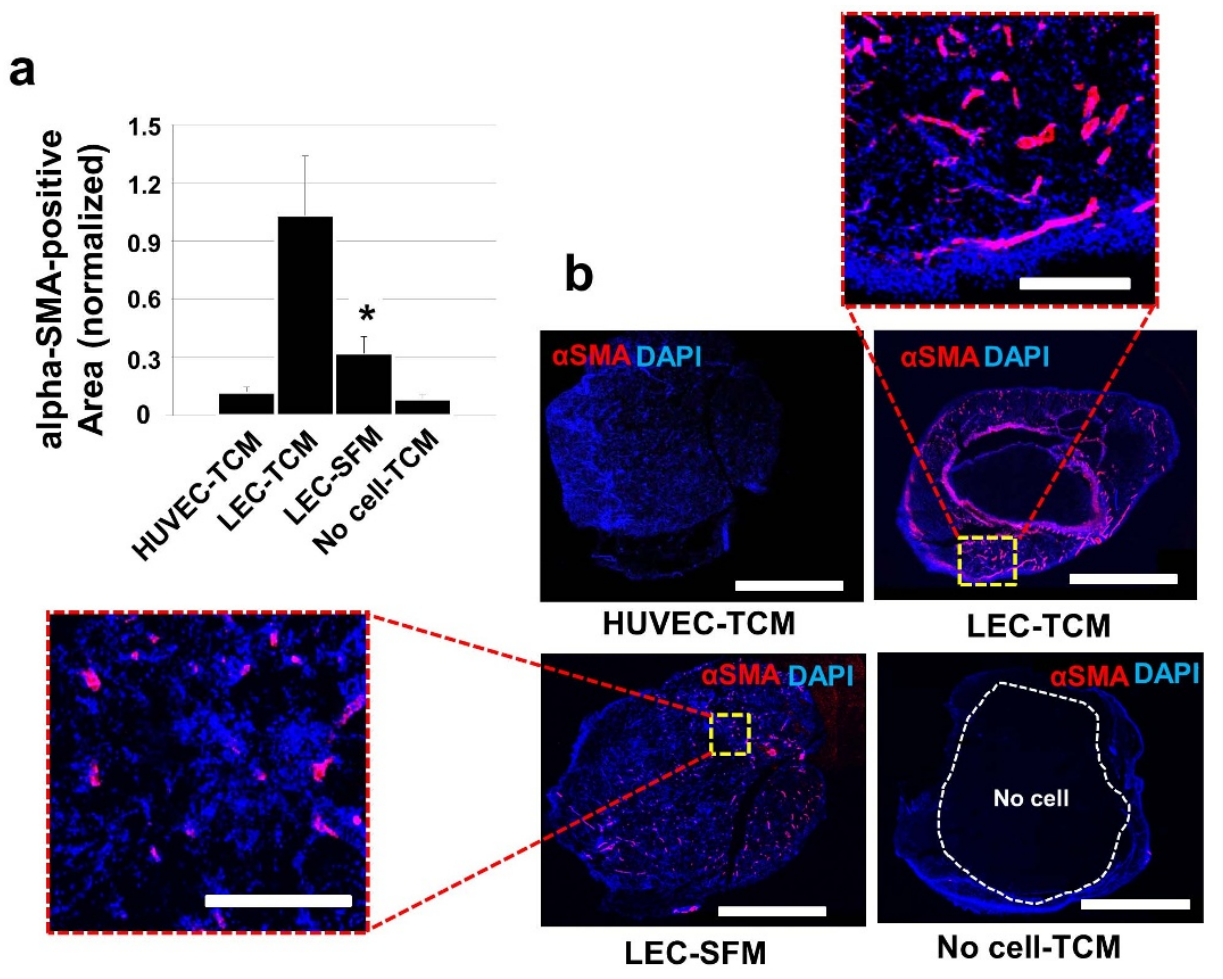

C
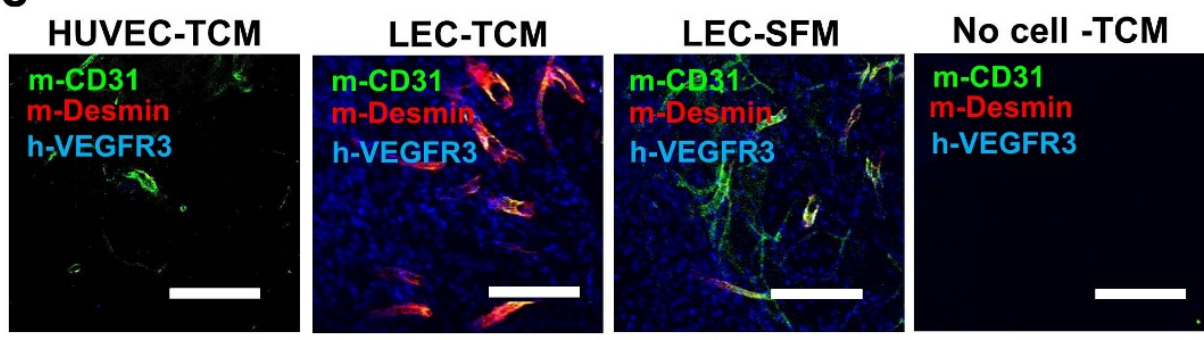

Figure 4 MB231-LEC recruit pericytes in vivo. High-concentrate matrigel containing LEC or HUVEC $\left(2 \times 10^{6} /\right.$ gel $)$ and heparin $(10$ units/gel $)$ was injected subcutaneously. TCM or SFM ( $50 \mu \mathrm{l}$ /injection) was subcutaneously dosed daily for 10 days, the mice were euthanized, and the gel plugs were excised and analyzed. (a) Gel plugs were sectioned and probed with anti-alpha-SMA ( $\alpha$ SMA) antibodies. $\alpha$ SMA positive cells per 12 randomly selected areas were quantified by using ImageJ. The LEC-TCM group shows significant infiltration of the $\alpha$ SMA positive cells, compared to the LEC-SFM group $\left({ }^{*} \mathrm{P}=0.031\right)$. (b) Representative confocal images for $\alpha$ SMA-positive area in four different groups of plugs (HUVEC-TCM, LEC-TCM, LEC-SFM, No cell-TCM). Scale bars in the whole images and in the enlarged images represent 5,000 $\mu \mathrm{m}$ and 1,000 $\mu \mathrm{m}$, respectively. (c) To confirm the pericyte infiltration and blood vessel coverages by the pericytes, four different plug samples were co-stained with anti-desmin and anti-mCD31 antibodies. LECincluded matrigel plugs in animals treated with TCM showed higher coverage of the blood vessels by pericytes, compared to TCM-treated HUVEC, SFMtreated LEC, and TCM-treated no cell groups. Anti-hVEGFR3 antibodies (blue) were used to detect human LEC that are included in the gel plugs. Only LEC-TCM and LEC-SFM group showed LEC. Scale bars represent $200 \mu \mathrm{m}$. Data (a) are reported as mean \pm s.e.m.

\section{Discussion}

The presence of LEC, a component of lymphatic endothelium in tumor microenvironment is a result of tumor lymphangiogenesis. Tumor lymphangiogenesis is driven by tumor cell-secreted lymphangiogenic growth factors such as VEGFC and VEGFD ${ }^{32}$. It is well established that lymphatic vasculatures in the tumor microenvironment serve as routes of tumor dissemination because of that lymphatic vessels are much more permeable compared to blood vessels ${ }^{33}$. We add a new concept of the important role of LEC-secreted proteins (also called lymphangiocrine factors) and their signals in tumor microenvironment to the classical understanding of tumor lymphangiogenesis. We discovered that tumor-associated (or tumor-educated) LEC express EGF and PDGF-BB and influence the tumor microenvironment. We employed LEC-included MB231 tumor xenograft models with 2,000,000 LEC and 300,000 MB231 cells (Fig. 1a,b). We have designed our in vivo model based on previous studies $^{24-27}$. As primary human endothelial cells do not survive for a long time after subcutaneous injection in a mouse model, many of previous studies have employed a high number of endothelial cells. For example, Franses et al. injected 1,000,000 EC adjacent to 12 day old primary lung tumors ${ }^{24}$. Neiva et al. co-injected EC and cancer cells with the $900,000: 100,000$ ratios into mice ${ }^{25}$. Other stromal cells have also been co-injected with cancer cells. Suzuki et al co-injected mesenchymal stem cells (MSC) and cancer cells with the $2,500,000: 500,000(5: 1)$ ratios $^{26}$. Fridman et al. summarized very diverse ratios between cancer cell $(250,000$ to $4,000,000)$ and stromal cells, including EC, fibroblasts, MSC, bone marrow derived stromal cells $(74,000$ to $5,000,000)$ for the co-injection models ${ }^{27}$. Using our breast tumor xenograft model, we showed that LEC-included MB231 tumors exhibit a rapid tumor growth rate compared to normal MB231 tumors or MEC/HUVEC-included MB231 tumors (Fig. 1a,b). This might be related to LEC-secreted EGF (Fig. 2) and its enhancement of tumor cell proliferation (Fig. 3). The role of EGF in tumor cell proliferation has been well studied in many types of 

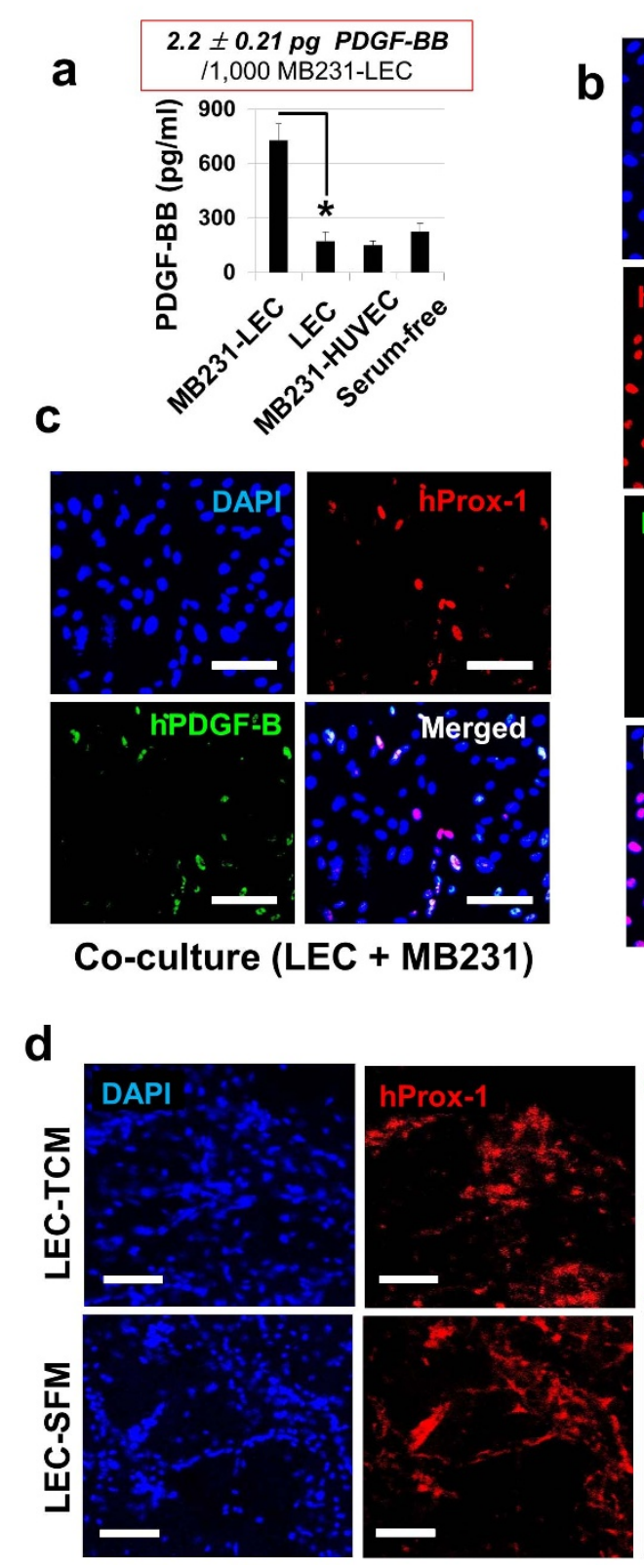



Monoculture (LEC)



Figure 5 | MB231-LEC express PDGF-BB. (a) ELISAs for PDGF-BB on MB231-LEC conditioned media, normal LEC CM, MB231-HUVEC CM, and serum-free media (SFM). MB231-LEC express high amount of PDGF-BB, compared to normal LEC ( $\left.{ }^{*} \mathrm{P}=0.026\right)$. We estimate that 1,000 MB231-LEC cells expressed $2.2 \pm 0.21 \mathrm{pg}$ hPDGF-BB. (b) Normal LEC in normal EGM media ('LEC') or LEC in TCM ('MB231-LEC') show distinct hPDGF-B expression. Normal LEC do not express PDGF-B, but TCM conditioned LEC express PDGF-B. Scale bars represent $100 \mu \mathrm{m}$. (c) LEC and MB231 were cocultured in a same plate. In the co-culture condition, LEC start to express PDGF-B, however MB231 do not show PDGF-B expression. Scale bars represent $100 \mu \mathrm{m}$. (d) LEC-included matrigel plug assay. Matrigel containing LEC $\left(2 \times 10^{6} /\right.$ gel $)$ and heparin (10 units/gel) was injected subcutaneously. TCM or SFM (50 $\mu \mathrm{l} /$ injection) was subcutaneously dosed daily for 10 days, the mice were euthanized, and the gel plugs were excised and analyzed. Human Prox-1 positive hLEC in the matrigel plugs express human PDGF-B (hPDGF-B) when the animals are treated with TCM ('LEC-TCM' group). hLEC from SFMtreated animals do not express PDGF-B ('LEC-SFM' group). Scale bars represent $100 \mu \mathrm{m}$. Data (a) are reported as mean \pm s.e.m.

cancer using experimental and computational approaches ${ }^{34,35}$. The role of the host (mouse) LEC in EGF-driven tumor growth versus the injected hLEC could be assessed by the application of anti-human EGF or anti-mouse EGF antibodies which would specifically block hEGF or mEGF. These experiments would address the possible clinical relevance of LEC-expressed EGF in patients. Moreover, the mechanisms of EGF expression in tumor-educated LEC need to be further elucidated. Although many of studies have previously focused on the mechanism of EGF expression in cancer cells ${ }^{36}$, stud- ies to determine the mechanism of EGF secretion by LEC have not been done.

MB231-LEC also express PDGF-BB and may influence tumor microenvironment (Fig. 4,5,6). PDGF-BB is involved in tumor growth, pericyte coverage, angiogenesis, and tumor drug resistance ${ }^{37-39}$. Increased pericyte infiltration and angiogenesis by MB231-LEC (Fig. 4) was observed, and this can be explained by LEC-secreted PDGF-BB (Fig. 5), and the inhibition of the PDGFR $\beta$ signaling pathway with SU16f (a PDGFR $\beta$ inhibitor) 
a

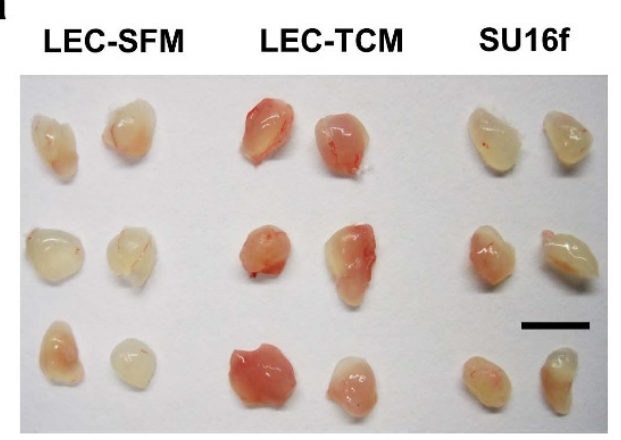

C LEC-SFM
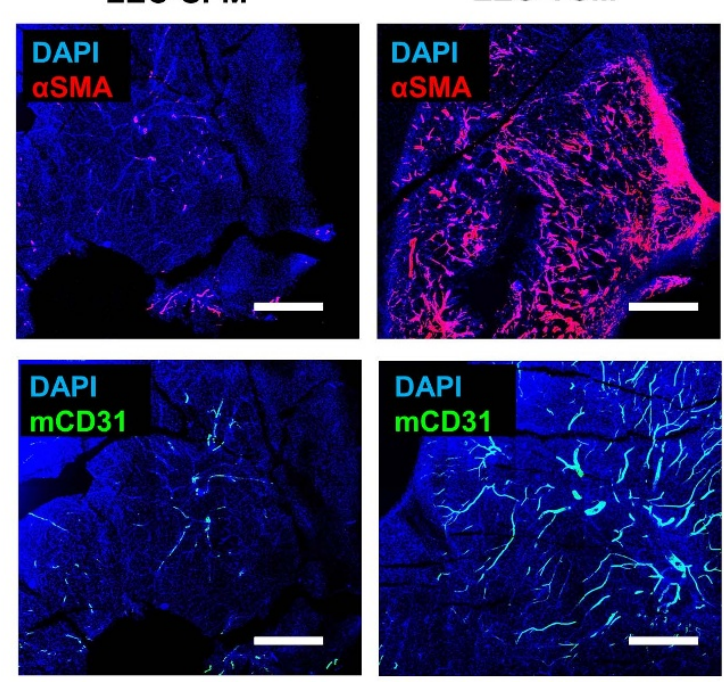

b




microenvironment contains apoptotic molecules. This idea is quite attractive because death of pericytes is closely related to blood vessel mediated metastasis ${ }^{43,44}$ and tumors may set the balance between adequate perfusion and metastasis by differentially regulating pericyte apoptosis. These hypotheses need to be further tested. However, regardless of mechanisms, the data showing apoptosis-experiencing pericytes suggest that PDGF-BB secreted by tumor-educated LEC (MB231-LEC) may influence pericyte recruitment and maintenance in the tumor microenvironment.

Interestingly, MEC-included MB231 tumors showed suppression of tumor growth. Currently, the role of blood endothelial cells (BEC) in tumor growth is quite controversial. It has been reported that BEC block tumor growth ${ }^{24}$. In that study, perlecan from BEC muted the proliferative and invasive phenotype of lung and breast cancer cells in vitro. However, perlecan silencing in BEC significantly increased IL6 secretion in BEC, eliminating the ability of the BEC to inhibit cancer cell invasiveness. In contrast, other studies have reported that $\mathrm{BEC}$ induce tumor growth via cancer stem cell related mechanisms and tumorigenic crosstalk with tumor microenvironment ${ }^{14,45,46}$. In the present MB231 model, HUVEC did not affect tumor growth, but MEC suppressed tumor growth, suggesting that different BEC lines could result in different phenotypes. Interestingly, MEC has a distinct secretome; CD26, GM-CSF, and HB-EGF are highly expressed. HB-EGF is also highly expressed by MB231 cells as described in our previous study ${ }^{29}$, so the contribution of the HB-EGF secreted by MEC to tumor growth is likely to be minimal. CD26 and GM-CSF have been linked to tumor inhibitory mechanisms. CD26 (also called DPPIV) inhibitors extended GLP-2 mediated tumor promoting effects on intestinal cancer cells ${ }^{47}$. CD26 inhibited the malignant phenotype of prostate cancer cells by blocking the basic fibroblast growth factor signaling pathway ${ }^{48}$. GM-CSF inhibited breast cancer growth and metastasis by invoking an anti-angiogenic program in tumor-educated macrophages ${ }^{49}$. GM-CSF also involved in antitumor immunity ${ }^{50,51}$. Therefore, the role of MEC secreted factors in tumor growth needs to be further investigated.

In summary, lymphangiocrine factors derived from LEC may support tumor growth by promoting tumor cell proliferation, pericyte infiltration, and angiogenesis whereas MEC promote tumor suppression. This study shows for the first time the diverse roles that different EC can play in the breast tumor microenvironment.

\section{Methods}

Cell culture. Human umbilical vein endothelial cells (HUVEC), lymphatic endothelial cells (LEC), and microvascular endothelial cells (MEC) were purchased from Lonza, and grown in EGM-2 (HUVEC) and EGM-2MV (LEC and MEC). MDA-MB-231 breast cancer cells were provided by Dr. Zaver Bhujwalla (JHMI, Radiology and Oncology). MDA-MB-231 cells were propagated in RPMI-1640 medium supplemented with $10 \%$ FBS and $1 \%$ penicillin/streptomycin (Sigma).

Conditioned media. When MDA-MB-231 cells reached confluence in T175 tissue culture flasks, the normal growth media was replaced with $8 \mathrm{ml}$ of serum-free media (SFM). After $24 \mathrm{~h}$ incubation, the supernatant was centrifuged and filtered through $0.2 \mu \mathrm{m}$ syringe filters (Corning). The resulting tumor-conditioned media (TCM) was stored in aliquots at $-80^{\circ} \mathrm{C}$. When LEC/HUVEC/MEC reached $30 \%$ confluence in T75 tissue culture flasks, the growth media (EGM) was replaced with $30 \%$ TCM (TCM:EGM $=3: 7$ ) to allow the TCM to educate the LEC/HUVEC/MEC. LEC/ HUVEC/MEC were allowed to grow in the media for 4 days then the media was replaced with $3 \mathrm{ml}$ SFM containing 2\% FBS (not supplemented with bullet kit). After $48 \mathrm{~h}$, the supernatant was centrifuged and filtered. The resulting tumor-educated LEC/HUVEC/MEC conditioned media (MB231-LEC or MB231-HUVEC or MB231MEC) was stored in aliquots at $-80^{\circ} \mathrm{C}$ to avoid multiple freeze thaws.

LEC-included tumor xenograft models. To evaluate roles of the various endothelial cells (EC) in the tumor microenvironment, a relatively small number of MDA-MB231 cells $\left(3 \times 10^{5} /\right.$ mouse, $100 \mu \mathrm{l}$ of RPMI- 1640 complete media with no matrigel) were orthotopically inoculated into the thoracic mammary fat pad of the athymic nude mice (female, 5-6 weeks) with or without two million LEC, MEC, or HUVEC. This system was used to maximize the contribution of EC and to evaluate the roles of these EC in tumor growth and the tumor microenvironment. The tumor size was measured by using a caliper, and the volume was calculated, using the formula: $\mathrm{V}=$ $0.52 \times$ (tumor length) $\times(\text { tumor width })^{2}$.
Reverse western blot assays. For reverse western blot, Proteome Profiler Antibody Array Kits for human angiogenesis factors (R\&D Systems) was used, according to the manufacturer's instructions. Factors present in LEC, MEC, or HUVEC conditioned media were compared with those in conditioned media from tumor-educated LEC (MB231-LEC), MB231-MEC, or MB231-HUVEC. Average signal (pixel density) of the pair of duplicate spots was determined using ImageJ. Expression level changes more than $200 \%$ after TCM treatment were considered as a significant perturbation. We further studied these highly changed target molecules (e.g., EGF and PDGF-BB in LEC).

LEC-included matrigel plug assay. High-concentrate matrigel $(500 \mu \mathrm{l}, \mathrm{BD}$ Biosciences) containing LEC or HUVEC $\left(2 \times 10^{6} /\right.$ gel $)$ and heparin (10 units/gel) was injected subcutaneously on the ventral side of both flanks of athymic nude mice. TCM or SFM ( $50 \mu \mathrm{l} /$ injection) was subcutaneously administered daily for 10 days, the mice were euthanized, and the gel plugs were excised and analyzed. Evaluating role of LECexpressed PDGF-BB in pericyte recruitment and angiogenesis, SU16f, a selective PDGFR $\beta$ inhibitor was employed in the same LEC-included matrigel plug assays. There were three groups: 'LEC-SFM' group (LEC-matrigel injection and SFM treatment for 10 days); 'LEC-TCM' group (LEC-matrigel injection and TCM treatment for 10 days); and 'SU16f' group (LEC-matrigel injection and TCM/SU16f co-treatment for 10 days). TCM and SFM were injected subcutaneously. SU16f was dissolved in 10\% DMSO solution (PBS base) and orally administered using an oral gavage for mouse feeding ( $10 \mathrm{mg} / \mathrm{kg} / \mathrm{day}$ ). Two control groups ('LEC-TCM' and 'LEC-SFM' groups) were also orally treated with vehicle (10\% DMSO in PBS).

Immunofluorescence. We performed immunofluorescence with tumor tissues, matrigel plugs, and mono- or co-cultured cells. Tumor tissues and matrigel plugs, fixed in $10 \%$ neutral buffered formalin (Sigma) overnight, were placed in $30 \%$ sucrose (Sigma) in PBS, incubated overnight at $4^{\circ} \mathrm{C}$, and frozen in the O.C.T. compound (Sakura). Sections of $10-\mu \mathrm{m}$ thickness were cut at $-20^{\circ} \mathrm{C}$. To perform immunostaining with monocultured LEC, 25,000 LEC in normal media were plated in an 8-well Tissue-Tek and the cells were incubated overnight. The normal media was replaced with control media (30\% SFM $+70 \%$ EGM) or $30 \%$ TCM media $(30 \%$ $\mathrm{TCM}+70 \% \mathrm{EGM})$, then the plate was incubated overnight. In LEC/MB231 coculture model, we mixed 10,000 LEC and 10,000 MB231 (1:1) in 50\% EGM and 50\% RPMI-1640 media and make the cell adhere on the bottom for overnight. We then replaced the co-media with serum-free media and incubated the cells overnight again Mono- or co-cultured cells were fixed in $10 \%$ neutral buffered formalin for $1 \mathrm{~h}$ at room temperature (RT) before immunostaining.

After blocking with 5\% normal goat or normal chicken serum (Jackson Immunoresearch) in PBST ( $0.3 \%$ Triton) for $1 \mathrm{~h}$ at room temperature (RT), the tissue sections or cells were treated with one or more of the following primary antibodies overnight at $4{ }^{\circ} \mathrm{C}$ : goat anti-human VEGFR3 (1:200, R \& D systems), rabbit antiProx-1 antibody ( $1: 500$, AngioBio), rat anti-mouse CD31 ( $1: 100$, BD Pharmingen), mouse anti-human EGF ( $1: 200$, Santa Cruz), mouse anti-human PDGF-B $(1: 200$, Santa Cruz), rat anti-human PCNA ( $1: 1000$, BD Pharmingen), goat anti-mouse desmin (Santa-Cruz), mouse anti-smooth muscle actin Cy-3 (SMA, 1:500, Sigma), rabbit anti-pEGFR (Cell Signaling), rabbit anti-cleaved caspase 3 (1:500, Cell Signaling), and goat anti-mouse lectin FITC ( $1: 100$, Sigma). After 3 rinses with PBST, sections were incubated for 1 hour at RT with one or more of the following secondary antibodies $(1: 500)$ : FITC-conjugated goat anti-rat, FITC-conjugated chicken antigoat, rhodamine-conjugated goat anti-rat, $\mathrm{Cy} 3$-conjugated goat anti-rabbit, Alexa Fluor 488 goat anti-mouse, FITC goat anti-rabbit, DyLight 405 goat anti-rabbit, and DyLight405 goat anti-mouse antibodies (all from Jackson Immunoresearch). After 3 rinses with PBST, the samples were counterstained with DAPI $(1: 10,000$, Roche) ( 5 min at RT). The samples were washed with PBST once and mounted with the ProLong Gold anti-fade reagent (Invitrogen) in the dark. Fluorescent signals were visualized and digital images were obtained using the LSM-510 confocal microscope (Carl Zeiss).

Real Time PCR. LEC (700,000 cells per flask) were plated in $100 \mathrm{~mm}$ tissue culture flasks in normal EGM media. After one day when they are attached on the bottom of the flask, the normal media was replaced with experimental control media (70\% EGM $+30 \%$ SFM) or TCM media (70\% EGM + 30\% TCM), and cells were further incubated for overnight. Total RNA was extracted from normal LEC or tumoreducated LEC by the JHU Core using Qiagen's RNeasy Plus Mini Kit. Briefly, LEC were lysed, and disrupted with a QIAshredder spin column prior to putting over a special column for removal of genomic DNA. RNA was bound to second spin column for RNA extraction, washed several times, then RNA was eluted with RNase-free water. To prepare cDNA, normal LEC or tumor-educated LEC derived RNA $(0.5 \mu \mathrm{g}$ per group) was mixed with $4 \mu \mathrm{l}$ of $5 \times$ iScript reaction mix (BioRad), $1 \mu \mathrm{l}$ of iScript reverse transcriptase (BioRad), and nuclease-free water making total volume of $20 \mu \mathrm{l}$. cDNA synthesis was performed by the iQ5 multicolor RT-PCR Detection System (BioRad). Gathered $20 \mu \mathrm{l}$ cDNA was 5 times more diluted with nuclease-free water for next RT-PCR. $5 \mu \mathrm{l}$ of SYBR, $1 \mu \mathrm{l}$ of primer (described below), $1 \mu \mathrm{l}$ of cDNA (5 times diluted), and $3 \mu \mathrm{l}$ nuclease-free water were mixed, and the RT-PCR was carried out using the CFX96 Real-Time System and C1000 Touch Thermal Cycler. We used BioRad Prime PCR assays for given target genes, human PDGFB, and human GAPDH.

The amplicon context sequence of the human PDGFB primers is AGCTCGCCTCCAGAGTGGGAGCGGGTCATGTTCAGGTCCAACTCGGCCCCATC 
TTCCTCTCCGGGGTCTCCGTGCAGCAGGCGTTGGAGATCATCAAAGGAGCGGA TCGAGTGGTC (assay ID: qHsaCID0016004, BioRad).

The amplicon context sequence of the human GAPDH primer is

GTATGACAACGAATTTGGCTACAGCAACAGGGTGGTGGACCTCATGGCCCACAT GGCCTCCAAGGAGTAAGACCCCTGGACCACCAGCCCCAGCAAGAGCACAAGAG GAAGAGAGAGACCCTCACTGCTGGGGAGTCCCTGCCACAC (assay ID: qHsaCED0038674, BioRad).

Cell proliferation assays. Proliferation assays were performed by using the WST-1 reagent (Roche) as previously described ${ }^{52}$. WST-1 is reduced by mitochondrial dehydrogenases and transformed into dark yellow colored Formazan. MDA-MB-231 cells were seeded in 96-well plates at a density of 1,500 cells/well in $100 \mu \mathrm{l}$ RPMI-1640 media. The plates were incubated overnight, then the RPMI-1640 media was replaced with LEC or HUVEC conditioned media (LEC-TCM, LEC-SFM, HUVEC-TCM) or serum-free media (SFM). Anti-EGFR antibodies (R\&D systems) were employed in the LEC conditioned media to inhibit LEC-derived EGF effects in cancer cell proliferation. The plates were incubated for $72 \mathrm{~h}$, after which the conditioned media (with or without anti-EGFR antibodies) was removed and $100 \mu \mathrm{l}$ of WST-1 solution ( $1: 10$ dilution of WST-1 in SFM) was added. After incubation for $4 \mathrm{~h}$, the absorbance at $450 \mathrm{~nm}$ was measured using the Victor V plate reader (Perkin Elmer).

Statistical Analysis. Error bars correspond to s.e.m, unless otherwise stated. Differences between two groups are regarded as significant when $\mathrm{P}$ is less than 0.05 using the Student's t-test.

1. Kim, Y., Stolarska, M. A. \& Othmer, H. G. The role of the microenvironment in tumor growth and invasion. Prog Biophys Mol Biol 106, 353-379 (2011).

2. Polyak, K., Haviv, I. \& Campbell, I. G. Co-evolution of tumor cells and their microenvironment. Trends Genet 25, 30-38 (2009).

3. Mukhtar, R. A., Nseyo, O., Campbell, M. J. \& Esserman, L. J. Tumor-associated macrophages in breast cancer as potential biomarkers for new treatments and diagnostics. Expert Rev Mol Diagn 11, 91-100 (2011).

4. Yu, Y. et al. Cancer-associated fibroblasts induce epithelial-mesenchymal transition of breast cancer cells through paracrine TGF-beta signalling. $\mathrm{Br} \mathrm{J}$ Cancer 110, 724-732 (2014)

5. Cirri, P. \& Chiarugi, P. Cancer-associated-fibroblasts and tumour cells: a diabolic liaison driving cancer progression. Cancer Metastasis Rev 31, 195-208 (2012).

6. Jain, R. K. \& Carmeliet, P. SnapShot: Tumor angiogenesis. Cell 149, 1408-1408 e1401 (2012).

7. Raica, M. \& Ribatti, D. Targeting tumor lymphangiogenesis: an update. Curr Med Chem 17, 698-708 (2010).

8. Kerbel, R. S. Tumor angiogenesis. N Engl J Med 358, 2039-2049 (2008).

9. Butler, J. M., Kobayashi, H. \& Rafii, S. Instructive role of the vascular niche in promoting tumour growth and tissue repair by angiocrine factors. Nat Rev Cancer 10, 138-146 (2010).

10. Lu, J. et al. Endothelial cells promote the colorectal cancer stem cell phenotype through a soluble form of Jagged-1. Cancer Cell 23, 171-185 (2013).

11. Franses, J. W. \& Edelman, E. R. The evolution of endothelial regulatory paradigms in cancer biology and vascular repair. Cancer Res 71, 7339-7344 (2011).

12. Krishnamurthy, S. et al. Endothelial cell-initiated signaling promotes the survival and self-renewal of cancer stem cells. Cancer Res 70, 9969-9978 (2010).

13. St Croix, B. et al. Genes expressed in human tumor endothelium. Science 289, 1197-1202 (2000).

14. Warner, K. A. et al. Endothelial cells enhance tumor cell invasion through a crosstalk mediated by CXC chemokine signaling. Neoplasia 10, 131-139 (2008).

15. Zeng, Q. et al. Crosstalk between tumor and endothelial cells promotes tumor angiogenesis by MAPK activation of Notch signaling. Cancer Cell 8, 13-23 (2005).

16. Cols, M. et al. Stromal endothelial cells establish a bidirectional crosstalk with chronic lymphocytic leukemia cells through the TNF-related factors BAFF, APRIL, and CD40L. J Immunol 188, 6071-6083 (2012).

17. Alitalo, A. \& Detmar, M. Interaction of tumor cells and lymphatic vessels in cancer progression. Oncogene 31, 4499-4508 (2012).

18. Kim, U., Park, H. C. \& Choi, K. H. Differential permeability of lymphatic and blood vessels in determining the route of metastasis as demonstrated by indirect lymphography. Clin Exp Metastasis 6, 291-299 (1988).

19. Moskowitz, M. et al. Breast cancer screening. Preliminary report of 207 biopsies performed in 4, 128 volunteer screenees. Cancer 36, 2245-2250 (1975).

20. Ran, S., Volk, L., Hall, K. \& Flister, M. J. Lymphangiogenesis and lymphatic metastasis in breast cancer. Pathophysiology 17, 229-251 (2010).

21. Hirakawa, S. et al. Identification of vascular lineage-specific genes by transcriptional profiling of isolated blood vascular and lymphatic endothelial cells. Am J Pathol 162, 575-586 (2003).

22. Alitalo, K. The lymphatic vasculature in disease. Nat Med 17, 1371-1380 (2011)

23. Lee, E., Pandey, N. B. \& Popel, A. S. Pre-treatment of mice with tumorconditioned media accelerates metastasis to lymph nodes and lungs: a new spontaneous breast cancer metastasis model. Clin Exp Metastasis 31, 67-79 (2014).

24. Franses, J. W., Baker, A. B., Chitalia, V. C. \& Edelman, E. R. Stromal endothelial cells directly influence cancer progression. Sci Transl Med 3, 66ra65 (2011).

25. Neiva, K. G. et al. Endothelial cell-derived interleukin-6 regulates tumor growth. BMC Cancer 14, 99 (2014).
26. Suzuki, K. et al. Mesenchymal stromal cells promote tumor growth through the enhancement of neovascularization. Mol Med 17, 579-587 (2011).

27. Fridman, R., Benton, G., Aranoutova, I., Kleinman, H. K. \& Bonfil, R. D. Increased initiation and growth of tumor cell lines, cancer stem cells and biopsy material in mice using basement membrane matrix protein (Cultrex or Matrigel) coinjection. Nat Protoc 7, 1138-1144 (2012).

28. Fridman, R. et al. Enhanced tumor growth of both primary and established human and murine tumor cells in athymic mice after coinjection with Matrigel. J Natl Cancer Inst 83, 769-774 (1991).

29. Lee, E., Koskimaki, J. E., Pandey, N. B. \& Popel, A. S. Inhibition of lymphangiogenesis and angiogenesis in breast tumor xenografts and lymph nodes by a peptide derived from transmembrane protein 45 A. Neoplasia 15, 112-124 (2013).

30. Nico, B. et al. Desmin-positive pericytes in the chick embryo chorioallantoic membrane in response to fibroblast growth factor-2. Microvasc Res 68, 13-19 (2004).

31. Skalli, O. et al. Alpha-smooth muscle actin, a differentiation marker of smooth muscle cells, is present in microfilamentous bundles of pericytes. J Histochem Cytochem 37, 315-321 (1989).

32. Skobe, M. et al. Induction of tumor lymphangiogenesis by VEGF-C promotes breast cancer metastasis. Nat Med 7, 192-198 (2001).

33. Mayerson, H. S., Patterson, R. M., Mc, K. A., Lebrie, S. J. \& Mayerson, P. Permeability of lymphatic vessels. Am J Physiol 203, 98-106 (1962).

34. Ozawa, S. et al. Stimulation by EGF of the growth of EGF receptorhyperproducing tumor cells in athymic mice. Int J Cancer 40, 706-710 (1987).

35. Athale, C. A. \& Deisboeck, T. S. The effects of EGF-receptor density on multiscale tumor growth patterns. J Theor Biol 238, 771-779 (2006).

36. Shima, I. et al. Expression of epidermal growth-factor (EGF), matrix metalloproteinase- $9(\mathrm{mmp}-9)$ and proliferating cell nuclear antigen (pcna) in esophageal cancer. Int J Oncol 6, 833-839 (1995).

37. Greco, C. et al. c-MYC deregulation is involved in melphalan resistance of multiple myeloma: role of PDGF-BB. Int J Immunopathol Pharmacol 19, 67-79 (2006).

38. Xue, Y. et al. PDGF-BB modulates hematopoiesis and tumor angiogenesis by inducing erythropoietin production in stromal cells. Nat Med 18, 100-110 (2012).

39. Nissen, L. J. et al. Angiogenic factors FGF2 and PDGF-BB synergistically promote murine tumor neovascularization and metastasis. J Clin Invest 117, 2766-2777 (2007).

40. Cao, Y. Opinion: emerging mechanisms of tumour lymphangiogenesis and lymphatic metastasis. Nat Rev Cancer 5, 735-743 (2005).

41. Baranski, J. D. et al. Geometric control of vascular networks to enhance engineered tissue integration and function. Proc Natl Acad Sci U S A 110, 7586-7591 (2013).

42. He, W. et al. Pericyte-based human tissue engineered vascular grafts. Biomaterials 31, 8235-8244 (2010)

43. Garcia-Roman, J. \& Zentella-Dehesa, A. Vascular permeability changes involved in tumor metastasis. Cancer Lett 335, 259-269 (2013).

44. Xian, X. et al. Pericytes limit tumor cell metastasis. J Clin Invest 116, 642-651 (2006).

45. Kamenova, B. et al. Effective treatment of the brachial plexus syndrome in breast cancer patients by early detection and control of loco-regional metastases with radiation or systemic therapy. Int J Clin Oncol 14, 219-224 (2009).

46. Karl, E. et al. Unidirectional crosstalk between Bcl-xL and Bcl-2 enhances the angiogenic phenotype of endothelial cells. Cell Death Differ 14, 1657-1666 (2007).

47. Masur, K., Schwartz, F., Entschladen, F., Niggemann, B. \& Zaenker, K. S. DPPIV inhibitors extend GLP-2 mediated tumour promoting effects on intestinal cancer cells. Regul Pept 137, 147-155 (2006).

48. Wesley, U. V., McGroarty, M. \& Homoyouni, A. Dipeptidyl peptidase inhibits malignant phenotype of prostate cancer cells by blocking basic fibroblast growth factor signaling pathway. Cancer Res 65, 1325-1334 (2005).

49. Eubank, T. D. et al. Granulocyte macrophage colony-stimulating factor inhibits breast cancer growth and metastasis by invoking an anti-angiogenic program in tumor-educated macrophages. Cancer Res 69, 2133-2140 (2009).

50. Borrello, I. M. et al. Granulocyte-macrophage colony-stimulating factor (GMCSF)-secreting cellular immunotherapy in combination with autologous stem cell transplantation (ASCT) as postremission therapy for acute myeloid leukemia (AML). Blood 114, 1736-1745 (2009).

51. Emens, L. A. GM-CSF-secreting vaccines for solid tumors. Curr Opin Investig Drugs 10, 1315-1324 (2009).

52. Lee, E., Rosca, E. V., Pandey, N. B. \& Popel, A. S. Small peptides derived from somatotropin domain-containing proteins inhibit blood and lymphatic endothelial cell proliferation, migration, adhesion and tube formation. Int $J$ Biochem Cell Biol 43, 1812-1821 (2011).

\section{Acknowledgments}

We thank Dr. Zaver Bhujwalla for providing MDA-MB-231 breast cancer cells. We are grateful to Dr. Farhad Vesuna and Ashley Irving for their help in RT-PCR experiments. This work was supported by the grants from the National Institutes of Health R01 CA138264 and the Safeway Foundation. 


\section{Author contributions}

E.L., N.B.P. and A.S.P. conceived the work. E.L. performed most of the experiments. E.L. N.B.P. and A.S.P. interpreted the experimental data. E.L. wrote the manuscript, and E.L., N.B.P. and A.S.P. edited the manuscript.

\section{Additional information}

Supplementary information accompanies this paper at http://www.nature.com/ scientificreports

Competing financial interests: The authors declare no competing financial interests.
How to cite this article: Lee, E., Pandey, N.B. \& Popel, A.S. Lymphatic endothelial cells support tumor growth in breast cancer. Sci. Rep. 4, 5853; DOI:10.1038/srep05853 (2014)

(c) (i) (5) (2) This work is licensed under a Creative Commons Attribution-NonCommercialShareAlike 4.0 International License. The images or other third party material in this article are included in the article's Creative Commons license, unless indicated otherwise in the credit line; if the material is not included under the Creative Commons license, users will need to obtain permission from the license holder in order to reproduce the material. To view a copy of this license, visit http:// creativecommons.org/licenses/by-nc-sa/4.0/ 